\title{
The Microscopic Structure and Adsorption Performance of Amide Ions on the Mica (001) Surface
}

\author{
Dan Su, ${ }^{1, a}$, Yuelong $\mathrm{Liu}^{1, \mathrm{~b},{ }^{*}}$ and Gousheng $\mathrm{Liu}^{2, \mathrm{c}}$ \\ ${ }^{1}$ School of Chemistry and Chemical Engineering, Jiangxi Science \& Technology Normal University, \\ Nanchang, Jiangxi 330013, China \\ ${ }^{2}$ State Key Laboratory of Chemical Engineering, East China University of Science and Technology, \\ Shanghai 200237, China \\ asuzihan1234@126.com, *,blylgyx@163.com, 'gsliu@ecust.edu.cn \\ * corresponding author: Yuelong Liu
}

Keywords: Molecular dynamics, Amide, Mica, Electrostatic attraction, Hydrogen bond.

Abstract. Molecular dynamics simulation has been empolyed to investigate microscopic structure and the adsorption performance of amide ions on the mica (001) surface in the vacuum. The results drawn from the computed interaction energies show that amides are more effective collectors for mica compared with quaternary amines. In addition to strong electrostatic attractions, the head group $\mathrm{H}$ atoms of the adsorbed amide ions can form hydrogen bonds to the bridging oxygen atoms on the mica surface.

\section{Introduction}

In recent years, with the rapid development of computer technology, computer simulation has become a bridge between microscopic world and macroscopic experiments, which helps us investigate some phenomena that cannot be completely understood in other ways. As a useful tool to simulate the abstract models of many natural systems, molecular dynamics simulation is widely used for studying the molecular structure and microscopic observation of interaction phenomena[1]. Wang et al.[2] studied the adsorption mechanism of sodium oleate ( $\mathrm{NaOL}$ ), dodecylamine hydrochloride (DDAH)and the mixture of them on the muscovite surface in the aqueous solution by means of molecular dynamics simulation. Rai et al.[3] used the molecular dynamics simulations to investigate adsorption mechanism of oleate and dodecylammonium chloride molecules on two different mineral surfaces, and computed the surface-surfactant interaction energies. The results based on theoretical computations matched remarkably well with the observed experiments. Wang et al.[4] revealed the state of adsorbed dodecylamine at a silica surface as a function of $\mathrm{pH}$ by experimental methods and molecular dynamics simulations.

In this study, in order to illustrate the fundamental problem of the interaction between amide ions with different chain lengths and the mica (001) surface, MD simulations are performed using PCFF_phyllosilicate force field [5]. The interaction energies of these surfactants on the mica surface are computed to quantify the interaction strength.

\section{Simulation details}

Models. The $5 \times 3 \times 1$ supercell model of mica is prepared according to ${ }^{29} \mathrm{Si} \mathrm{NMR}$ data. The double layer mica surface is built by cleaving the (001) surface at the middle of the interlayer. Models of the amide ions with alkyl chain lengths $\mathrm{n}=6,8,10, \ldots, 22$ are optimized using Dmol3 module and placed over the mica (001) surface by the "build Layer" tool. A large vacuum ( thickness ca. $80 \AA$ ) is added above the collector layer to eliminate the effect of periodic boundary conditions.

Simulations. After a short energy minimization with 100-steps, the NVT simulations are conducted using the Forcite package with the PCFF_phyllosilicate force field at the temperature of $298 \mathrm{~K}$ which is controlled by the Nose-Hoover-langevin (NHL) thermostat. The electrostatic interactions are described by the Ewald method, and the non-bond cutoff distance for van der Waals 
interactions is set to $12.5 \AA$, Total simulations are carried out with $3.0 \mathrm{~ns}$, and the production of the last $0.5 \mathrm{~ns}$ are used for data analysis.

\section{Results and Discussion}

Interaction energy. The interaction energy can be used to quantify the strength of the regent-mineral surface interaction [3] and is calculated using the following equation:

$$
\mathrm{E}_{\text {int eraction }}=\mathrm{E}_{\text {total }}-\left(\mathrm{E}_{\text {surface }}+\mathrm{E}_{\text {regent }}\right) \text {. }
$$

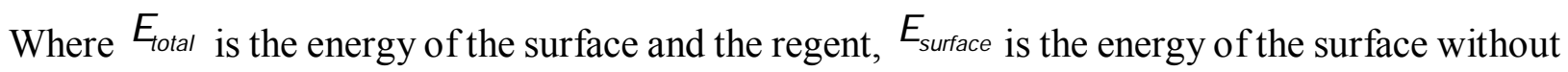
the regent and $E_{\text {regent }}$ is the energy of the regent without the surface.

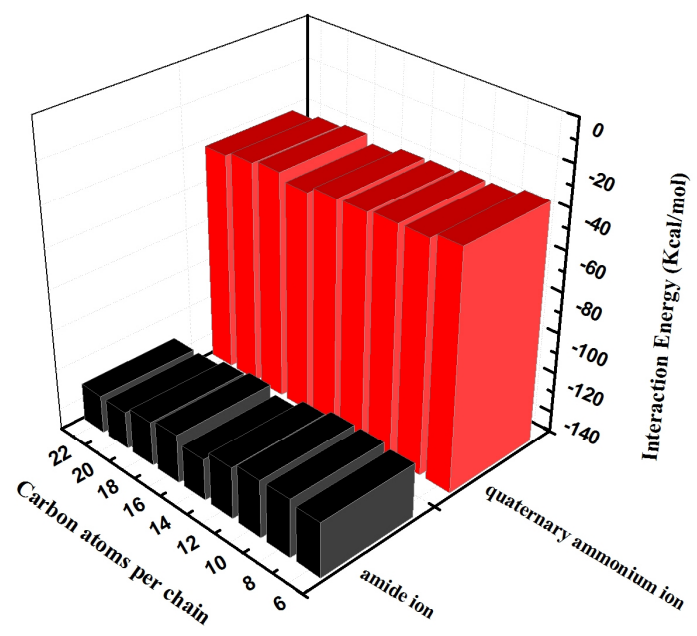

Fig. 1. Computed interaction energies bewteen amide ions and the mica surface

As illustrated in Fig.1, the calculated interaction energies of amide ions with mica surface are lower than that of quaternary ammoniums on the mica surface[6], indicating the stronger interactions between amide ions and the mica surface. Therefore, compared with quaternary amine, amide is much more effective collector for mica flotation, which is in good agreement with the results drawn from the flotation experiments of these cationic collectors onto mica in the earlier work [7].

Adsorption mechanism. The ESP charges of $\mathrm{NH}_{3}$ head groups of amide ions with alkyl chain lengths $6,8,10, \ldots, 22$ are shown in Fig. 2.

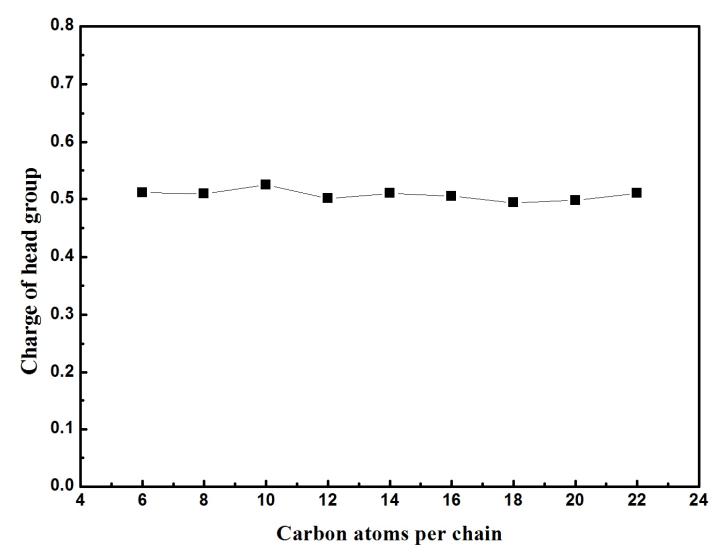

Fig. 2. The ESP charges of $\mathrm{NH}_{3}$ head groups of amide ions with different chain lengths 
As illustrated in Fig. 2, the calculated values are all positive and have almost no differences, indicating that the $\mathrm{N}$-containing polar head groups can adsorb on negatively charged mica surface and the effect of alkyl chain length on the atomic charge distribution of the surfactant cations is limited.

For a system, the total energy consists of valence, non-bond, and crossterm interactions, which can be mathematically expressed using the following equation:

$$
\mathrm{E}_{\text {total }}=\mathrm{E}_{\text {valence }}+\mathrm{E}_{\text {non-bond }}+\mathrm{E}_{\text {crossterm }} \text {. }
$$

As shown in Fig. 3, both total valence energy and van der Waals energy are positive. However, the value of electrostatic energy is negative, suggesting the great contribution of electrostatic energy to total energy. This means that the electrostatic attractions between positively charged head groups of amide ions and negatively charged mica surface is the driving force for the interaction of amide on the mica surface.

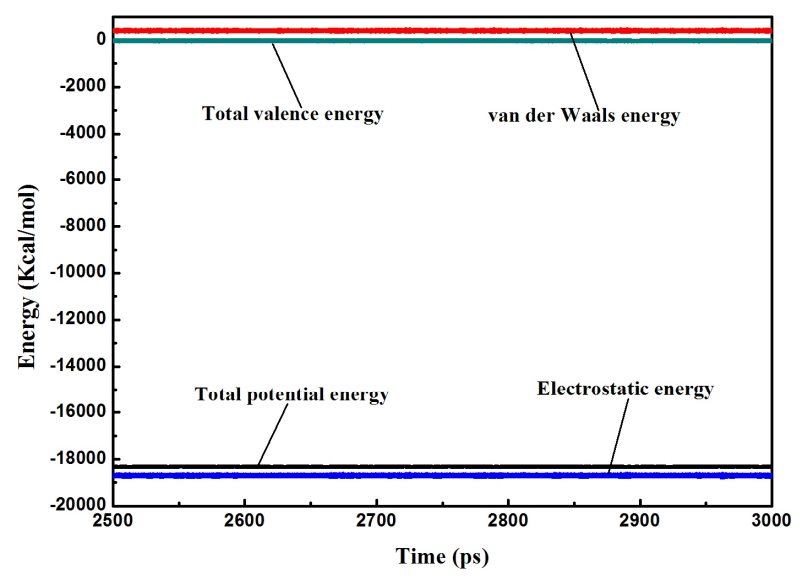

Fig. 3.Time evolution of energy of amide-mica system in equilibrium period

Hydrogen bond profile. To explore whether there is the existence of hydrogen-bonding interactions in these systems, radial distribution function (RDF) is analyzed. Radial distribution function (also sometimes referred to as pair correlation function) can be expressed using the following formula:

$g_{\alpha-\beta}(r)=\frac{1}{4 \pi \rho_{\beta} r^{2}} \frac{d N_{\alpha-\beta}}{d r}$

Where the $\rho_{\beta}$ denotes the number density of particle $\beta$ and the $N_{\alpha-\beta}$ denotes number of particles of the specified type.

For all amide-mica systems, the shapes and positions of peaks in the calculated RDFs kept similar and a representative result is shown in Fig.4.

We can observe three distinct peaks in Fig. 4 and the first peak appears at about $1.5 \AA$. This means that head groups of amide ions can form hydrogen bonds with the bridging oxygen atoms on mica surface. Besides electrostatic interaction, hydrogen bonding plays an important role in enhancing the strength of the amide-mica surface interaction. N-containing head groups of amide ions can closely approach the mica surface and show a configuration where the angle between the the $\alpha-\mathrm{C}-\mathrm{N}$ bond and the surface is about $66^{\circ}$, due to the formation of hydrogen bonds. 


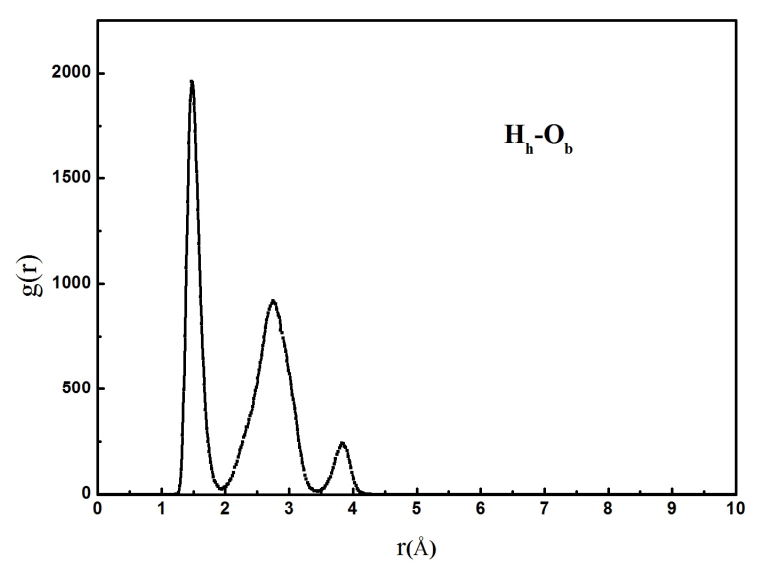

Fig. 4. RDF between head group $\mathrm{H}$ atoms of amide ion and surface bridging oxygen atoms

\section{Conclusions}

In this paper, the interaction between amide ions and the mica surface in the vacuum has been analyzed on the basis of molecular dynamics simulations using the PCFF_phyllosilicate force field. The adsorption energies of amide ions with different alkyl chain lengths on the mica (001) surface are calculated. Results reveal that amide is much more effective collector for mica than quaternary amine. By analyzing the potential energy and radial distribution function in our simulation, it is found that amide ions can adsorb on the mica surface by electrostatic attraction and hydrogen bonding.

\section{Acknowledgements}

This work was sponsored by the National Science Foundation of China (No. 51164009).

\section{References}

[1] Y.Xu, Y. L.Liu, S.Gao, Z.W.Jiang, D.Su and G. S.Liu: Chemical Engineering Science.Vol. 114 (2014), p. 58

[2] L.Wang, Y.Hu, W.Sun and Y. Sun: Applied Surface Science.Vol. 327 (2015), p. 364

[3] B.Rai, P.Sathish, J.Tanwar, K.Moon and D. Fuerstenau: Journal of Colloid and Interface Science. Vol. 362 (2011), p. 510

[4] X.M.Wang, J.Liu, H.Du and J.D.Miller:Langmuir. Vol. 26 (2009), p. 3407

[5] H.Heinz, H.Koerner, K.L.Anderson, R.A.Vaia and B.Farmer: Chemistry of Materials. Vol. 17 (2005), p. 5658

[6] Y.Xu, Y.L. Liu, D.D. He and G.S.Liu:Minerals Engineering. Vol. 53(2013), p. 101

[7] Z.Y Gao: Journal of Bgrimm.Vol. 1(1992), p. 60 (In Chinese) 